\title{
Los estilos de aprendizaje Vark en estudiantes universitarios de las escuelas de negocios
}

\section{The Vark Learning Styles among University Students of Business Schools}

\author{
Jenny L. Espinoza-Poves \\ Universidad de San Martín de Porres, Lima, Perú \\ ORCID: https://orcid.org/0000-0002-3761-0721 \\ Walter A. Miranda-Vílchez \\ Universidad de San Martín de Porres, Lima, Perú \\ ORCID: https://orcid.org/0000-0002-9839-468X \\ Raquel Chafloque-Céspedes* \\ Universidad de San Martín de Porres, Lima, Perú \\ ORCID: https://orcid.org/0000-0003-0314-5603
}

Recibido 29-10-18 Revisado 01-12-18 Aprobado 13-03-19 En línea 02-04-19

*Correspondencia

Email: mchafloquec@usmp.pe
Citar como:

\footnotetext{
Espinoza-Poves, J., Miranda-Vílchez, W., \& ChafloqueCéspedes, R. (2019). Los estilos de aprendizaje Vark en estudiantes universitarios de las escuelas de negocios. Propósitos y Representaciones, 7(2), 384-414. doi: http://dx.doi.org/10.20511/pyr2019.v7n2.254
} 


\section{Resumen}

La presente investigación tuvo como objetivo conocer los estilos de aprendizaje en los estudiantes de las escuelas de negocios de una universidad privada del departamento de Lambayeque. Mediante una encuesta aplicada, prueba de estilos de aprendizaje VARK, a 218 universitarios/as (71 estudiantes de Administración y 147 estudiantes de Administración de Negocios Internacionales), del II al X ciclo de las escuelas de negocios de dicha universidad durante el primer semestre del 2018. Se encontró que el $25.5 \%$ de los/as estudiantes tienen un estilo de aprendizaje multimodal; asimismo, se halló que la edad está asociada significativamente a los estilos de aprendizaje. Finalmente, se demuestra una relación significativa del estilo de aprendizaje y la escuela profesional, de un lado los/las estudiantes de Administración tienen en su mayoría un estilo de aprendizaje multimodal (dos o más estilos); mientras que los/las estudiantes de Negocios Internacionales tienen un estilo de aprendizaje lecto/escritora.

Palabras clave: Estilos de aprendizaje; VARK; Aprendizaje multimodal; Aprendizaje lecto/escritora; Estudiantes de pregrado.

\section{Summary}

The aim of this research was to know the learning styles in the students of the business schools of a private university in the Department of Lambayeque. An applied survey, a test of VARK learning styles, was applied to 218 university students (71 students of Administration and 147 students of International Business Administration), from the II to X term of the business schools of said University. During the first semester of the 2018, it was found that $25.5 \%$ of students have a multimodal learning style. It was also found that age is significantly associated with learning styles. Finally, a significant relationship between the learning style and the professional school is demonstrated. On the one hand the Administration students have mostly a multimodal learning style (two or more styles), while international business students have a reading/writing learning style.

Keywords: Learning Styles; VARK, Multimodal Learning; Reading/Writing Learning; Undergraduate.

\section{Introducción}

En el sector educativo terciario, específicamente en las universidades, la academia realiza de manera constante aportes para potenciar las capacidades de los estudiantes, para la mejora de su rendimiento académico. Las investigaciones abordan desde diagnósticos para saber los problemas externos e internos que influyen en el rendimiento académico (Chilca, 2017; Gul, et al., 2016; Chafloque, et al., 2018) y en temas de cómo mejorar el rendimiento académico ya sea mediante hábitos de estudio (Espinoza, 2017; Hernández, Rodríguez \& Vargas, 2012; Otero \& Torres, 2005), metodología de enseñanza (Palazón, Gómez, Cándido, Concepción, \& Gómez, 2011; Gonzalez \& María, 2007), ambientes y escenarios para un mejor aprendizaje, (García - Valcárcel \& Tejedor, s.f.), estilos de aprendizaje (Estrada, 2018; Freiberg, Ledesma \& Fernández, 2017; Acevedo, Tirado \& Montero, 2015; Ortiz, Sánchez \& Lozano, 2013; Esguerra \& Guerrero, 2010; Gonzáles, Constanza, \& Padilla, 2010) y entorno familiar (Torres \& Rodríguez, 2006; Covadonga, 2001).

Al concluir una clase o sesión de aprendizaje, el docente realiza una evaluación de los temas realizados, ya sea por medio de preguntas, exámenes o rubricas, el docente tendrá en cuenta diferentes criterios de acuerdo con la competencia que desea alcanzar (López, et al., 2014). A pesar de que la sesión de clase se desarrolla en condiciones similares para el grupo de estudiantes (mismo centro de estudio, misma escuela profesional, similares prerrequisitos adquiridos, similar capacidad intelectual, similar edad, mismo docente), los resultados de las evaluaciones evidencian que no todos los estudiantes logran el aprendizaje de manera satisfactoria. Esto no indica que un 
estudiante tenga menos capacidades que otros, sino que existen diferentes estilos de aprendizaje (Martín-García, 2003; Barrio \& Nicasio, 2000; Cano, 2000) y que cada estudiante tiene diferentes capacidades o que éstas pueden ir variando conforme pasa el tiempo (Gómez del Valle, 2003; Ordoñez, Rodriguez, \& Plaza, 2003).

El aprendizaje involucra una serie de "procesos biológicos y psicológicos que ocurren en la corteza cerebral, que gracias a la mediatización del pensamiento" (Díaz, 2012, pág. 6), el estudiante termina modificando la información como parte de su conocimiento, habilidad, actitud y experiencias que va adquiriendo al momento de interaccionar con el medio externo. Es decir, se puede hablar de aprendizaje cuando existe un cambio duradero, para lo cual la fase del hacer es importante.

Kolb (1975), toma como base la teoría de Piaget, concibiendo al aprendizaje en cuatro etapas que se van a dar como un espiral continuo y que de cumplirse todas, el tema de aprendizaje termina siendo significativo para el estudiante.

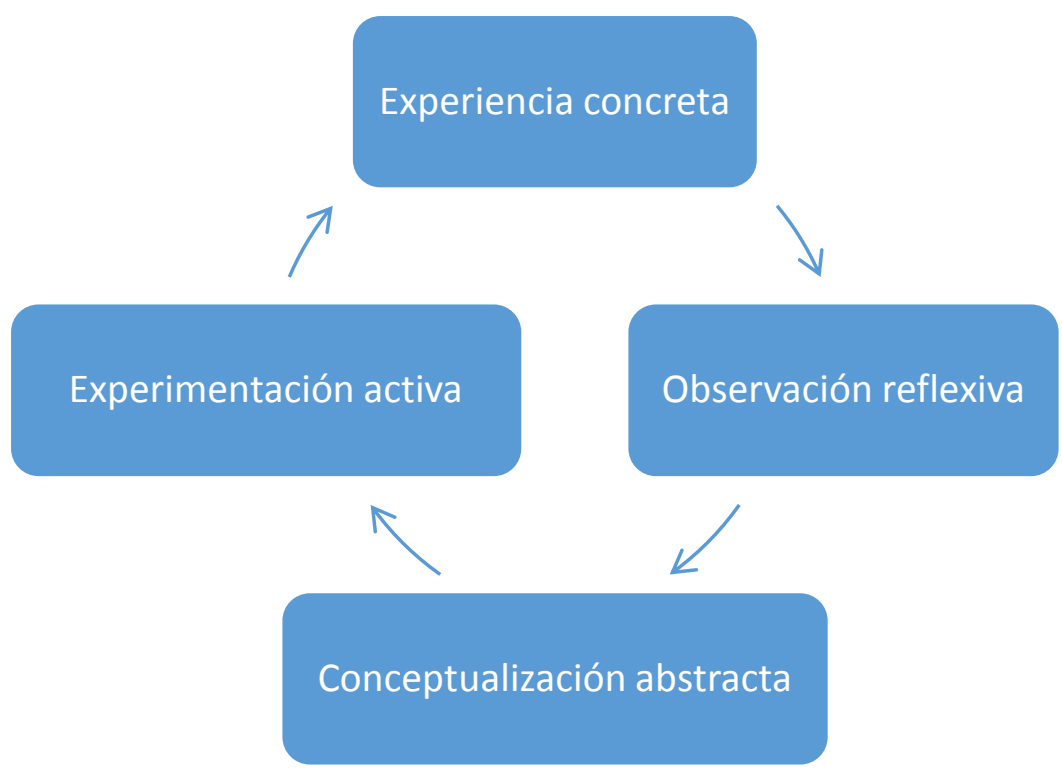

Figura 1. Etapas del ciclo de aprendizaje según David Kolb (Fuente: Diaz, 2012)

Cada estudiante, dependiendo de sus circunstancias particulares, interés, preferencia o gusto, se va a sentir más cómodo en alguna de las etapas, tal es así que si un estudiante aprende y disfruta de momentos que incluyen actividades formadoras de experiencias, es considerado como un estudiante activo. Por otro lado, tenemos a los estudiantes que se apasionan a través de la observación y en medio de ella reflexionan, éstos son considerados los estudiantes reflexivos. Otro grupo de estudiantes prefiere las fases donde se comparte el saber a través de conceptos, teorías, leyes, etc. a este grupo se le considera como estudiantes teóricos. Finalmente, están los estudiantes que gustan más de los momentos donde se aprende haciendo, este grupo de estudiantes son considerados como los pragmáticos (Diaz, 2012).

Richard Bandler y Jhon Grinder, proponen postulados que contribuyen a la Programación Neurolingüística (PNL), lo cual se fundamenta en que la persona tiende a tener un comportamiento producido y programable, donde el estado emocional va a estar determinado por las percepciones sensoriales, evidenciándose a través de una comunicación que puede ser verbal y no verbal (Vinyamata, 2011; Bórquez, 2002). La PNL termina agrupando en tres sistemas a las percepciones sensoriales: visual, auditivo, kinestésico.

En el presente trabajo, utilizamos el modelo propuesto por Neil Fleming y Collen Mills, quienes terminan en el 2006 de desarrollar un instrumento sencillo a fin de determinar las 
predilecciones de modalidad sensorial al momento de procesar información, al que denominan VARK: Visual, Auditivo, lector - escritor y kinestésico (García Nájera, 2007). Para Lozano (2004), el modelo VARK supone que cada estudiante puede identificar su propio estilo de aprendizaje, ser consciente de sus preferencias sensoriales (Sarmiento, Mayté, \& Tuyub, 2017), adecuarse al estilo de enseñanza del docente y actuar sobre su propia modalidad, con tal de aumentar el aprovechamiento en su aprendizaje (Núñez, Hernández, Tomás, \& Felipe, 2013). El modelo VARK proporciona una cuantificación de predilecciones de los estudiantes en cada una de los cuatro modos sensoriales (Gonzáles, Alonso, \& Rangel, 2012). En la Figura 2, se muestran actividades que pueden emplearse y que apoyarían cada estilo de aprendizaje.

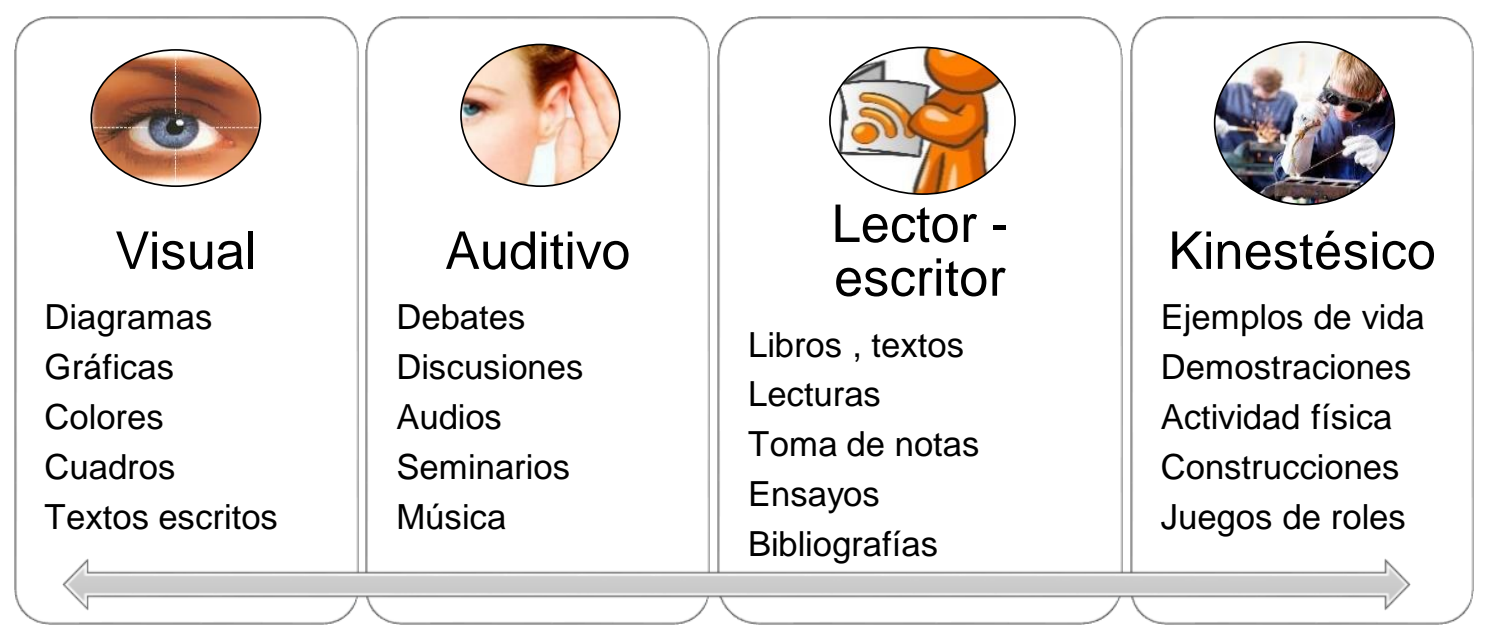

Figura 2. Actividades propuestas para cada estilo de aprendizaje VARK. (Fuente: Hawk \& Shah, 2007)

Muchas personas van a tener un sistema de representación dominante o en algunos casos llegar a combinar dos sistemas. Entonces, estando formada un aula por un grupo de estudiantes con características similares y cada uno de ellos con un distinto sistema de representación sensorial dominante, el docente deberá incorporar estrategias metodologías que beneficien a todos los estilos de aprendizaje, desde el momento de la motivación hasta la parte de evaluación de los saberes aprendidos (Varela, 2006).

En este contexto y teniendo en cuenta que existen diversos estilos de aprendizaje en los estudiantes universitarios, el presente estudio tiene como propósito examinar los estilos de aprendizaje de los estudiantes de pregrado de una universidad privada de la ciudad de Chiclayo.

El conocimiento generado a través de la presente investigación permitirá saber la relación existente entre las características académicas y demográficas, y los estilos de aprendizaje; así como comprender con mayor precisión los estilos de aprendizaje por escuelas profesionales. A partir de la evidencia generada, se podrá comprometer a directivos, docentes y estudiantes de la universidad en estudio, para que se comprometan con implementación de acciones para mejorar los estilos de aprendizaje de acuerdo con la escuela profesional.

\section{Método}

\section{Diseño de investigación.}

El diseño de investigación utilizado fue no experimental, descriptivo - correlacional.

\section{Población y muestra.}

La población estuvo formada por todos los estudiantes (hombres y mujeres), que cursaban del II al X ciclo de las escuelas de negocios, en el ciclo 2018 - I, de una universidad privada en la ciudad 
de Chiclayo. Siendo la población de 135 estudiantes de la escuela profesional de administración y 251 estudiantes de la escuela profesional de administración de negocios internacionales.

El tipo de muestreo utilizado fue el probabilístico estratificado por escuela profesional, con un $97 \%$ de confianza y un 5\% de error, quedando 71 estudiantes de la escuela profesional de administración y 147 de la escuela profesional de administración de negocios internacionales.

Participaron 218 estudiantes, 134 mujeres con una media de edad de 20.87 (D.E. 2.03) y 80 hombres con una media de edad de 21.64 (D.E. 2.20). Los estudiantes encuestados pertenecían a las escuelas profesionales de administración y administración de negocios internacionales, del segundo al décimo ciclo, el periodo de estudio fue durante el semestre académico 2018 - 1. En la Tabla 1 se puede observar las características demográficas y académicas de los estudiantes encuestados.

Tabla 1.

Características demográficas y académicas de la muestra.

\begin{tabular}{|c|c|}
\hline Administración (71) & $\begin{array}{l}\text { Administración de negocios internacionales } \\
\qquad(147)\end{array}$ \\
\hline Sexo & Sexo \\
\hline Mujer $=57.7 \%$ & Mujer $=64.6 \%$ \\
\hline Hombre $=42.3 \%$ & Hombre $=35.4 \%$ \\
\hline Edad & Edad \\
\hline 21.17 (D.E. 2.26) & 21.00 (D.E. 2.06) \\
\hline Ocupación & Ocupación \\
\hline Solo estudia $=65.7 \%$ & Solo estudia $=75.5 \%$ \\
\hline Estudia y trabaja $=34.3 \%$ & Estudia y trabaja $=24.5 \%$ \\
\hline Año de ingreso a la universidad & Año de ingreso a la universidad \\
\hline 2014.66 (D.E. 1.69) & 2014.17 (D.E. 1.89) \\
\hline Ciclo matriculado & Ciclo matriculado \\
\hline 5.97 (D.E. 2.42 ) & 6.40 (D.E. 6.43 ) \\
\hline Cursos matriculados & Cursos matriculados \\
\hline 6.43 (D.E. 1.57 ) & 7.56 (D.E. 684$)$ \\
\hline Cursos matriculados & Cursos matriculados \\
\hline Cursos generales $=7.6 \%$ & Cursos generales $=4.1 \%$ \\
\hline Cursos introductorios $=28.8 \%$ & Cursos introductorios $=36.6 \%$ \\
\hline Cursos formativos $=45.5 \%$ & Cursos formativos $=28.3 \%$ \\
\hline Cursos finales $=18.2 \%$ & Cursos finales $=31.0 \%$ \\
\hline Promedio ponderado & Promedio ponderado \\
\hline 13.70 (D.E. 1.52) & 13.29 (D.E. 1.49 ) \\
\hline
\end{tabular}

\section{Instrumento.}

El estudio utilizó un cuestionario estructurado bajo el formato de autoreporte, el cual contiene preguntas de información demográfica y académica, así como la escala de estilo de aprendizaje.

Escala de estilos de aprendizaje: se empleó la prueba "El Modelo VARK", instrumento propuesto por Fleming \& Mills (1992), donde diseñaron un instrumento que tome en cuenta el modo en que 
las personas procesan información según la preferencia de modalidad sensorial que tienen cada individuo. Para Lozano (2004), el modelo VARK que en un inicio identificaba la vista, el oído y el movimiento como estilos de aprendizaje, menciona que son justamente éstos los que marcan los primeros aprendizajes de la persona desde que nace, para luego una vez que adquiere la habilidad lecto - escritora, entonces tiene un nuevo modo de adquirir y filtrar información. Tal es así que en un inicio el Modelo VARK incluía sólo 13 preguntas, posteriormente en el 2006, tiene un total 16 preguntas con 4 respuestas cada una (Fleming, 2006).

Traducido del idioma inglés al español, revisado por expertos en el área de educación en cuanto a su grado de pertinencia, inteligibilidad, definición y formulación sintáctica de cada pregunta (ver Anexo 1).

Autores como Gónzales, Alonso, \& Rangel (2012), Nuñez, Hernández, Tomás, \& Felipe (2013) Dania \& Marchisio(2013 - 2014) y Castillo \& Mendoza (2015) utilizan el modelo VARK, a fin de evaluar el estilo de aprendizaje en una población con similares características al de la presente investigación (estudiantes universitarios).

\section{Análisis estadístico.}

El procesamiento estadístico de los datos se realizó por medio del software estadístico SPSS 22.0 para Windows. Para determinar la relación entre las variables que caracterizan a los estudiantes de las escuelas profesionales de negocios se utilizó la prueba de independencia. Asimismo, se realizó un ANOVA con el fin de averiguar si había diferencias significativas en la edad promedio de los estudiantes por cada estilo de aprendizaje que tiene el estudiante.

\section{Validez y fiabilidad.}

Para el análisis de fiabilidad de la escala, se usó el coeficiente de confiabilidad del Alfa de Cronbach. La escala de estilos de aprendizaje $(\alpha=0.528)$, obtuvo un Alfa inferior al mínimo esperado $(\alpha=0.706)$ esto se debe a que la escala presenta datos excluyentes, esto no indica algún problema ya que los estilos de aprendizaje son diferentes y esta puede excluir a la otra.

El test VARK fue validado por Leite, Svinicki \& Yuying (2009) a través de un análisis factorial confirmatorio, obteniendo puntajes de $0.85 ; 0.82 ; 0.84$ y 0.77 para cada subescala considerándose como un test adecuado.

En cuanto al análisis de validez de constructo se realizó por medio del Análisis de Componentes Principales (ACP) con rotación Varimax. Los 16 ítems en estudio proporcionaron una solución factorial de 6 componentes con una varianza total de $52.53 \%$ de los datos originales.

\section{Procedimiento.}

La encuesta fue administrada y asistida presencialmente por profesionales capacitados. Los estudiantes fueron informados de los objetivos y alcances del estudio, se garantizó la confidencialidad y el anonimato. Su participación fue voluntaria, sin ninguna clase de incentivos académicos, económicos o de otro tipo. Los estudiantes completaron la encuesta durante horas de clase, el tiempo promedio fue de 15 minutos.

\section{Resultados}

El modelo VARK, toma en cuenta, cuatro estilos de aprendizajes: visual, auditivo, lector - escritor y kinestésico. El 29.5\% de los estudiantes percibe la información a través de palabras escritas y mostradas en separatas o libros. Mientras que el 19.3\% aprende haciendo o experimentando, por ejemplo, en laboratorios, o ejercicios aplicados. En similar porcentaje el 17.4\% de estudiantes aprende escuchando y hablando, o sea al momento de participar en las aulas, obtienen mayor 
aprendizaje. Finalmente, el $8.3 \%$ de los estudiantes perciben la información a través de imágenes que podrían incluir, figuras, símbolos, cuadros, diapositivas y otros. Para Fleming (2006), una persona puede presentar 1 o más estilos de aprendizaje; el $25.7 \%$ de los/as estudiantes de las escuelas profesionales de administración y administración de negocios internacionales, tienen un estilo de aprendizaje multimodal (ver Figura 3).

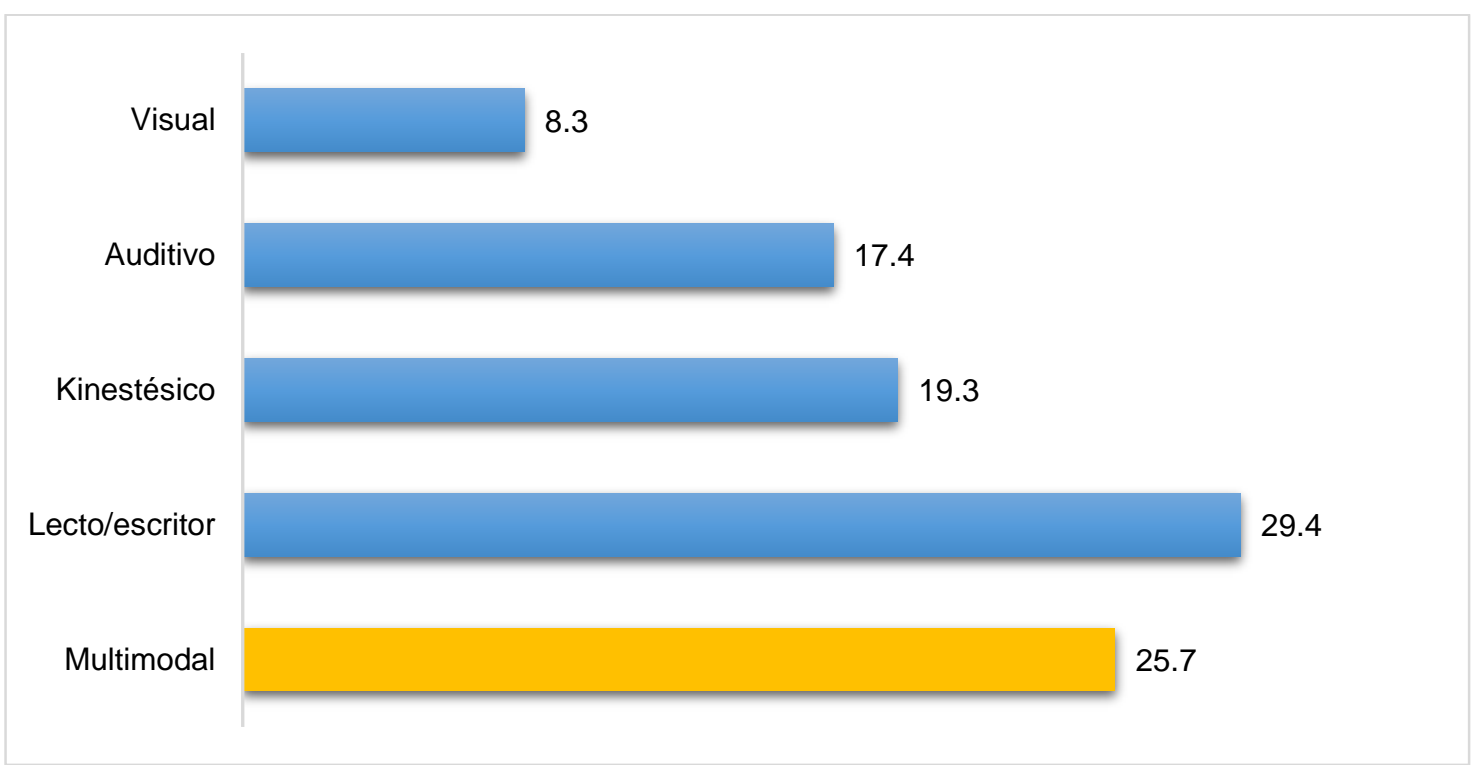

Figura 3. Estilos de aprendizaje de los estudiantes de las escuelas de negocios - VARK

La presente investigación, asocio las características socio demográficas y académicas con los estilos de aprendizaje, encontrando relaciones significativas con la edad y la escuela profesional. Según la edad, en los estudiantes de 18 años predomina el estilo de aprendizaje auditivo, en los estudiantes de 19 a 23 años el estilo de aprendizaje lecto/escritor, en los estudiantes de 24 años predomina el estilo de aprendizaje auditivo, y en los estudiantes que tienen entre 25 y 26 años el estilo de aprendizaje que aplican la mayoría de los estudiantes es kinestésico.

Otro aspecto importante analizado es el estilo de aprendizaje que destaca en cada estudiante tomando en cuenta la edad y el tipo de curso que lleva. En los estudiantes de 18 a 23 años el estilo de aprendizaje que predomina es el lecto/escritor especialmente en los estudiantes que llevan cursos generales, cursos introductorios, cursos de formación profesional y cursos de formación en investigación. 
Tabla 2.

Características de los estudiantes de las Escuelas de Negocios según estilos de aprendizaje.

\begin{tabular}{|c|c|c|c|c|c|}
\hline \multirow{2}{*}{ Características } & \multicolumn{5}{|c|}{ Estilos de aprendizaje } \\
\hline & V & A & $\mathrm{R}$ & $\mathrm{K}$ & Multimodal \\
\hline \multicolumn{6}{|l|}{ Sexo } \\
\hline Masculino & 22.2 & 33.3 & 43.1 & 45.2 & 33.9 \\
\hline Femenino & 77.8 & 66.7 & 56.9 & 54.8 & 66.1 \\
\hline \multicolumn{6}{|l|}{ Situación Académica } \\
\hline Sólo estudia & 73.2 & 59.5 & 75.0 & 83.3 & 72.6 \\
\hline Estudia y trabaja & 26.8 & 40.5 & 25.0 & 16.7 & 27.4 \\
\hline \multicolumn{6}{|l|}{ Escuela profesional* } \\
\hline Administración & 27.8 & 42.1 & 28.1 & 19.0 & 42.9 \\
\hline $\begin{array}{l}\text { Administración de Negocios } \\
\text { Internacionales }\end{array}$ & 72.2 & 57.9 & 71.9 & 81.0 & 57.1 \\
\hline \multicolumn{6}{|l|}{ Experiencia laboral } \\
\hline $\mathrm{Si}$ & 50.0 & 54.1 & 55.0 & 67.6 & 50.9 \\
\hline No & 50.0 & 45.9 & 50. & 32.4 & 49.1 \\
\hline \multicolumn{6}{|l|}{ Edad** } \\
\hline $18-20$ años & 38.9 & 45.9 & 39.7 & 41.5 & 32.1 \\
\hline $21-23$ años & 38.9 & 21.6 & 54.0 & 51.2 & 50.0 \\
\hline $24-26$ años & 22.2 & 32.4 & 6.3 & 7.3 & 17.9 \\
\hline \multicolumn{6}{|l|}{ Rendimiento Académico } \\
\hline Menos 11.9 & 11.8 & 10.5 & 9.5 & 9.5 & 47.3 \\
\hline $12-13.9$ & 35.3 & 42.1 & 58.7 & 58.7 & 14.5 \\
\hline Más 14 & 52.9 & 47.4 & 31.7 & 31.7 & 38.2 \\
\hline
\end{tabular}

Nota: *p-valor<.05, ** p-valor <.01

La presente investigación encontró una asociación significativa entre los estilos de aprendizaje y la escuela profesional. El $35.6 \%$ de estudiantes que pertenecen a la escuela profesional de Administración el tienen un estilo de aprendizaje multimodal (incluye 2 o más estilos de aprendizaje). Por otro lado, los estudiantes que pertenecen a la escuela profesional de 
Administración de Negocios Internacionales se inclinan al estilo de aprendizaje lecto/escritor $(32.0 \%)$ (ver Tabla 3$)$.

Tabla 3.

Estilos de aprendizaje según escuela profesional de negocios.

Escuela Profesional

\begin{tabular}{ccc} 
Estilo de Aprendizaje* & Administración de Negocios \\
\cline { 2 - 3 } & Anternacionales
\end{tabular}

\begin{tabular}{ccc}
\hline V & 6.8 & 8.7 \\
A & 21.9 & 14.7 \\
R & 24.7 & 32.0 \\
K & 11.0 & 23.3 \\
Multimodal & 35.6 & 21.3
\end{tabular}

*P-valor <.05

La malla curricular de las escuelas profesionales de negocios esta divida por áreas o tipo de formación que va adquiriendo el estudiante, dividiéndose en: cursos de formación generales, introductorios, profesional e investigación. En la Tabla 4 observamos, que el grupo de estudiantes que llevan cursos de formación general predomina el estilo de aprendizaje Kinestésico (31.6\%), aquellos que llevan cursos introductorios y de formación en investigación en su mayoría tienen un aprendizaje lecto / escritora (27.1\%). Finalmente, en el grupo de estudiantes que lleva cursos de formación profesional predomina el estilo de aprendizaje multimodal (34.7\%). A pesar de encontrar resultados evidentes, no se encontró una asociación significativa entre las variables.

Tabla 4.

Estilos de aprendizaje y tipo de curso.

\begin{tabular}{lccccc}
\hline \multirow{2}{*}{ Tipo de curso } & \multicolumn{5}{c}{ Estilo de Aprendizaje } \\
\cline { 2 - 6 } & $\mathrm{V}$ & $\mathrm{A}$ & $\mathrm{R}$ & $\mathrm{K}$ & Multimodal \\
\hline Formación en general & 9.1 & 18.2 & 27.3 & 36.4 & 9.1 \\
Formación en introductorio & 6.6 & 18.4 & 31.6 & 22.4 & 21.1 \\
Formación profesional & 5.6 & 15.3 & 31.9 & 12.5 & 34.7 \\
Formación en investigación & 13.6 & 18.6 & 27.1 & 18.6 & 22.0 \\
\hline
\end{tabular}

En la Tabla 4, se observa la relación entre el rendimiento académico y los estilos de aprendizaje, a pesar de no existir asociación significativa; podemos observar indicios que los estudiantes que tienen un rendimiento académico menor a 11.9 tiene un estilo de aprendizaje multimodal, mientras que los estudiantes que tienen un rendimiento académico de 12 a 13.9 tienen un aprendizaje lecto/escritor y finalmente los estudiantes que tienen rendimiento académico mayor a 14, predominan dos estilos de aprendizaje: multimodal y kinestésico. 
Tabla 5.

Estilos de aprendizaje y rendimiento académico.

\begin{tabular}{cccc}
\hline \multirow{2}{*}{$\begin{array}{c}\text { Estilo de } \\
\text { Aprendizaje }\end{array}$} & Menos de 11.99 & De 12-13.99 & Más de 14 \\
\cline { 2 - 4 } & 8.3 & 5.7 & 9.9 \\
V & 16.7 & 15.2 & 19.8 \\
A & 25.0 & 37.1 & 22.0 \\
R & 16.7 & 16.2 & 24.2 \\
K & 33.3 & 25.7 & 24.2 \\
Multimodal & &
\end{tabular}

A través de un análisis de varianza se probó que existen diferencias significativas en el estilo de aprendizaje y la edad del estudiante. El estilo de aprendizaje auditivo influirá más de acuerdo con la edad del estudiante, siendo los estudiantes de 24 años los que utilizan más este estilo de aprendizaje (F: 2.896, P-valor=0.004).

\section{Discusión}

Estudiar los estilos de aprendizaje ayuda a comprender el proceso de percibir y procesar la información, en este caso de los estudiantes de pregrado (Alonso, \& Rangel, 2012; Nuñez, Hernández, Tomás, \& Felipe, 2013; Dania \& Marchisio, 2013 - 2014; Castillo \& Mendoza, 2015). En el presente estudio, el $25.5 \%$ de los estudiantes tiene un estilo de aprendizaje multimodal; Varela (2006), menciona a Fleming, quien brinda un aporte significativo a la educación, al sugerir a los docentes en conocer y adaptar sus sesiones de aprendizaje a las preferencias que tienen sus estudiantes para recibir información y como consecuencia facilitarles el aprendizaje hacia un rendimiento óptimo.

La malla curricular de cada escuela profesional contiene una serie de cursos que van a contribuir a la formación profesional de los estudiantes según la especialidad que se haya elegido. Los estudiantes de la escuela profesional de Administración tienen en su mayoría un estilo de aprendizaje multimodal (dos o más estilos), propio de un administrador de empresas que deberá tener habilidades para organizar, investigar, analizar, proponer, ejecutar y tomar decisiones acertadas, teniendo visión integral, crítica e innovadora (FCARRHH, 2018). Por otro lado, los estudiantes de la escuela profesional de Negocios Internacionales tienen la mayoría un estilo de aprendizaje lector - escritor, propio de la formación que ellos estan adquiriendo donde para tomar decisiones necesitan conocer y tener una visión de la empresa en un contexto global, para lo cual debe leer acerca de la realidad exterior e interior de un país (FCARRHH, 2018). Nuñez, Hernández, Tomás, \& Felipe (2013) en la investigación que realizaron a los estudiantes de la escuela profesional de Computación, encuentran que éstos tienen un estilo de aprendizaje kinestésico, guardando relación con el desarrollo de la mayoría de sus cursos que involucra actividades prácticas, movimiento, tiempo, espacio, es decir aprenden a través del hacer. Es muy importante conocer los estilos de aprendizaje según la formación profesional que persiguen los estudiantes, a fin de que los cursos contenidos en la malla curricular utilicen técnicas de enseñanza, sistemas de evaluación y actividades que contribuyan a formar estudiantes competentes profesionalmente. 
En el presente estudio, el rendimiento académico no está asociado de manera significativa al estilo de aprendizaje, similar conclusión encontrada en otras investigaciones (Chafloque, et al., 2018; Acevedo, Tirado, \& Montero; 2015; Ortiz, Sánchez, \& Lozano, 2013; Gonzáles, Constanza, $\&$ Padilla, 2010) indicando que otros son los factores que influyen en el rendimiento académico (ambientales, motivacionales, físicos, emocionales, etc.). Sin embargo, es necesario conocer si los docentes están considerando el estilo de aprendizaje de los estudiantes para impartir sus sesiones de aprendizaje, de acuerdo a lo que sustenta Gónzales, Alonso, \& Rangel (2012).

La edad está asociada significativamente a los estilos de aprendizaje, en el grupo de estudiantes más jovenes (18 a 23 años) predomina en su mayoría un solo estilo de aprendizaje, en los estudiantes de 24 a 26 años predomina el estilo de aprendizaje multimodal. Martín \& Rodríguez (2003), mencionan que a medida que las personas van adquiriendo más edad pierde ese carácter (referiendose a estilo de aprendizaje) dominante, pues empiezan a combinar distintos estilos según sea el momento, lo que refuerza a su hipótesis de que a mayor edad en las personas existe variabilidad interindivual. Investigaciones como Gómez del Valle (2003) y Ordoñez, Rodriguez, \& Plaza, (2003) sugieren realizar un estudio longitudinal para ver si la edad tiene repercusion en la definición del estilo de aprendizaje.

El sexo y el estilo de aprendizaje no estan asociados de manera significativa, tal es asi que tanto varones como mujeres tienen un estilo de aprendizaje lecto/escritor y multimodal en su mayoría. Barrio \& Nicasio, (2000), Cano (2000) encuentran que existen diferencias en los estilos de aprendizaje de acuerdo al sexo del estudiante. En la misma linea, Martín-García (2003), explica que el estilo de aprendizaje no está asociado a variables sociodemográficas (sexo, estado civil y grado de instrucción).

De acuerdo a los objetivos trazados se logra describir los estilos de aprendizaje de los estudiantes de las Escuelas de Negocios, encontrando que de acuerdo a la escuela profesional, el estudiante tiene un estilo de aprendizaje marcado (Administración: Multimodal, Administración de Negocios Internacionales: Lecto/escritor), además no existe asociación significativa entre los estilos de aprendizaje y el rendimiento academico de los estudiantes, sexo, situación ocupacional, tipo de curso y experiencia profesional. Asimismo, se encontró asociación significativa entre el estilo de aprendizaje y la edad de los estudiantes, encontrando que a medida que avanza la edad el estilo de aprendizaje deja de ser uno sólo.

Finalmente es necesario estudiar otras variables como motivación, hábitos de estudios, ambientes para el aprendizaje, etc., para saber si las variables mencionadas al relacionarse con los estilos de aprendizaje contribuyen a la mejora del rendimiento académico.

\section{Referencias}

Acevedo, D., Tirado, D., \& Montero, P. (2015). Perfil de aprendizaje y rendimiento académico en una asignatura de química en modalidad distancia y presencial en dos programas de ingeniería. Formación Universitaria, 8(6), 39 - 45. Doi: https://dx.doi.org/10.4067/S071850062015000600006

Barrio, J., \& Nicasio, J. (2000). Diferencias en el estilo de aprendizaje. Psicothema, 12(2), 180 186. Recuperado de: http://www.psicothema.es/pdf/274.pdf

Bórquez, S. (2002). PNL: Tres letras para facilitar el cambio. PHAROS, 9(1), 75 - 91. Recuperado de: http://www.redalyc.org/html/208/20809106/

Cano, F. (2000). Diferencias de género en estrategias y estilos de aprendizaje. Psicothema, 12(3), 360 - 367. Recuperado de: http://www.psicothema.com/pdf/343.pdf

Cardozo, A. (2008). Motivación, aprendizaje y rendimiento académico en estudiantes del primer año universitario. Revista Laurus, 14(28), 209 - 237. Recuperado de: http://www.redalyc.org/pdf/761/76111716011.pdf 
Castillo, M., \& Mendoza, J. (2015). Estilos de aprendizaje en estudiantes universitarios: recursos informáticos como estrategia para su evaluación. Revista UNAH INNOV@ (4), 33 - 39. Doi: https://doi.org/10.5377/unahinnov.v0i4.2896

Chafloque-Céspedes, R., Vara-Horna, A., López-Odar, D., Santi-Huaranca, I., Diaz-Rosillo, A., \& Asencios-Gonzalez, Z. (2018). Ausentismo, presentismo y rendimiento académico en estudiantes de universidades peruanas. Propósitos y Representaciones, 6(1), 83-133. Doi: http:// dx.doi.org/10.20511/pyr2018.v6n1.177

Covadonga, d. (2001). Factores familiares vinculados al bajo rendimiento. Revista Complutense de Educación, 12(1), 81 - 113. Recuperado de: http://revistas.ucm.es/index.php/RCED/article/viewFile/RCED0101120081A/16850

Dania, C., \& Marchisio, S. (2013 - 2014). Dania, C., \& Roggiano, S. M. (2013). Modalidades de percepción sensorial de estudiantes de ingeniería en sistemas de información: aportes al diseño de material didáctico para la enseñanza de la Algoritmia. Invenio: Revista de Investigación académica (31), 215 - 228. Recuperado de https://dialnet.unirioja.es/servlet/articulo?codigo $=4776739$

Díaz, E. (2012). Estilos de Aprendizaje. Eídos, 5, 5-11. Recuperado de https://revistas.ute.edu.ec/index.php/eidos/article/view/88/81

Duart, J., \& Osorio, L. (2011). Análisis de la interacción en ambientes híbridos de aprendizaje. Revista Científica Iberoamericana de comunicación y educación, 37(2), 65 - 80. Doi: https://doi.org/10.3916/C37-2011-02-06

Esguerra, G., \& Guerrero, P. (2010). Estilos de aprendizaje y rendimiento académico en estudiantes de Psicología. Diversitas: Perspectivas en Psicología, 6(1), 97 - 109. Doi: https://doi.org/10.15332/s1794-9998.2010.0001.07

Espinoza, J. (2017). Relación entre los hábitos de estudio y el rendimiento académico de los alumnos de escuelas profesionales acreditadas. Tzhoecoen, 9(4), 29-40. Recuperado de: http://revistas.uss.edu.pe/index.php/tzh/article/view/721

Estrada, A. (2018). Estilos de aprendizaje y rendimiento académico. Revista Boletín Redipe, 7(7), 218 - 228. Recuperado de: https://revista.redipe.org/index.php/1/article/view/536

FCCAAyRRHH. (2018). Universidad San Martín de Porres - Facultad de Ciencias Administrativas $\quad \mathrm{y}$ Recursos Humanos: http://www.administracion.usmp.edu.pe/administracion/perfil-profesional/

FCCAAyRRHH. (2018). Universidad San Martín de Porres - Facultad de Ciencias Administrativas $\quad \mathrm{y}$ Recursos Humanos: http://www.administracion.usmp.edu.pe/negocios/perfil-profesional/

Fleming, N. (2006). A guide to learning styles. Recuperado de http://vark-learn.com/wpcontent/uploads/2014/08/The-VARK-Questionnaire-Spanish.pdf

Freiberg, A., Ledesma, R., \& Fernández, M. (2017). Estilos y estrategias de aprendizaje en estudiantes universitarios de Buenos Aires. Revista de Psicología, 35(2), 537 - 573. Doi: http://dx.doi.org/10.18800/psico.201702.006

García - Valcárcel, A., \& Tejedor, F. (s.f.). Variables TIC vinculadas a la generación de nuevos escenarios de aprendizaje en la enseñanza universitaria: aportes de las curvas roc para el análisis de diferencias. Educación XXI: revista de la Facultad de Educación, 14(2), 43 78. Doi: https://doi.org/10.5944/educxx1.14.2.237

García Nájera, J. (2007). El Modelo Vark: Instrumento diseñado para Identificar Estilos de Enseñanza - Aprendizaje. Investigación Educativa Duranguense, 6, 86-90. Recuperado de https://dialnet.unirioja.es/descarga/articulo/2293085.pdf

Gómez del Valle, M. (2003). Identificación de los estilos de aprendizaje predominantes en estudiantes de magisterio de la Facultad de Ciencias de la Educación de la Universidad de Cádiz. Revista Electrónica Interuniversitaria de Formación del Profesorado, 6(2). Recuperado de: https://dialnet.unirioja.es/servlet/articulo?codigo $=754490$

Gónzales, B., Alonso, C., \& Rangel, R. (2012). El modelo VARK y el diseño de cursos en línea. Revista mexicana de bachillerato a distancia, 4(8), 96 - 103. Recuperado de http://revistas.unam.mx/index.php/rmbd/article/view/44282/40032

Gonzáles, K., Constanza, N. \& Padilla, J. (2010). Incidencia del estilo de aprendizaje en el rendimiento académico en un curso virtual. Revista Virtual Universidad Católica del Norte, 
31 , 6

24.

Recuperado

de:

http://revistavirtual.ucn.edu.co/index.php/RevistaUCN/article/view/35/79

González, N., \& María, G. (2007). El aprendizaje cooperativo como estrategia de enseñanza aprendizaje en psicopedagogía (UC) repercusiones y valoraciones de los estudiantes. Revista Iberomericana de Educación, 42, 6 - 10. Recuperado de: https://dialnet.unirioja.es/servlet/articulo?codigo $=2283799$

Hawk, T., \& Shah, A. (2007). Using learning style instruments to enhance student learning. Decision Sciences of Innovative Education 5(1), 1 - 19. Recuperado de: http://citeseerx.ist.psu.edu/viewdoc/download?doi=10.1.1.470.4049\&rep=rep1\&type=pdf

Hernández, C., Rodríguez, N., \& Vargas, Á. (2012). Los hábitos de estudio y motivación para el aprendizaje de los alumnos en tres carreras de ingeniería en un tecnológico federal de la ciudad de México. Revista de la educación superior, 41(163), 67-87. Recuperado de: $\mathrm{http} / / / \mathrm{www}$.scielo.org.mx/pdf/resu/v41n163/v41n163a3.pdf

Lamas, H. (2008). Aprendizaje autorregulado, motivación y rendimiento académico. Liberabit, 14(14), 15 - 20. Recuperado de http://www.scielo.org.pe/pdf/liber/v14n14/a03v14n14.pdf

Leite, W., Svinicki, M., \& Yuying, S. (2009). Attempted Validation of the Scores of the VARK: Learning Styles Inventory with Multitrait -Multimethod Confirmatory Factor Analysis Models. Educational and Psychological Measurement, 70(2), 323 - 339. Doi: https://doi.org/10.1177/0013164409344507

López, J., Miribel, P., Colomer, J., Manuel, C., Sieiro, J., \& Ruiz, O. S. (2014). Evaluación de competencias mediante rúbricas en el área de la electrónica analógica. Revista del Congrés Internacional de Docència Universitària i Innovació (CIDUI), 2. Recuperado de https://www.cidui.org/revistacidui/index.php/cidui/article/view/537

Lozano, A. (2004). Estilos de enseñanza y aprendizaje. Un panorama de la estilística educativa. México: Trillas: ITESM.

Maquilón, J., \& Hernández, F. (2011). Influencia de la motivación en el rendimiento académico de los estudiantes de formación profesional. Revista electrónica interuniversitaria de formación del profesorado, 14(1). Recuperado de https://dialnet.unirioja.es/servlet/articulo?codigo $=3678771$

Martín - García, A. (2003). Estilos de aprendizaje en la vejez. Un estudio a la luz de la teoría del aprendizaje experiencial. Revista Española de Geriatría y Gerontología, 38(5), 258 - 265. doi: https://doi.org/10.1016/S0211-139X(03)74896-3

Martín, A., \& Rodríguez, M. (2003). Estilos de aprendizaje y grupos de edad. Aula Abierta, 82, 97 - 116. Recuperado de https://dialnet.unirioja.es/servlet/articulo?codigo=1012059

Núñez, F., Hernández, R., Tomás, V., \& Felipe, A. (2013). Identificación de estilos de aprendizaje en alumnos universitarios de computación de la Huasteca Hialguense mediante técnicas de minería de datos. Recuperado de: https://www.uaeh.edu.mx/investigacion/producto.php?producto=5566

Ordoñez, F., Rodríguez, R., \& Plaza, R. (2003). Análisis de los estilos de aprendizaje predominantes entre los estudiantes de ciencias de la salud. Eglobal, 2(2), 1-6. Doi: https://doi.org/10.6018/eglobal.2.2.619

Ortiz, E., Sánchez, A., \& Lozano, A. (2013). REA y estilos de aprendizaje según VARK en el aprendizaje de las matemáticas. Revista Internacional Magisterio: Educación y Pedagogía (64), 91 - 93. Recuperado de: http://catedra.ruv.itesm.mx/handle/987654321/773

Otero, V., \& Torres, L. (2005). Análisis de los hábitos de estudio en una muestra de alumnos universitarios. Revista Iberoamericana de Educación, 36(7), 1-9. Doi: https://rieoei.org/RIE/article/view/2929

Palazón, A., Gómez, M., Cándido, J., Concepción, M., \& Gómez, J. (2011). Relación entre la aplicación de metodologías docentes activas y el aprendizaje del estudiante universitario. Bordón, 63(2), 27-40. Recuperado de: https://recyt.fecyt.es/index.php/BORDON/article/view/28969

Sarmiento, A., Mayté, C., \& Tuyub, T. (2017). Identificación de los estilos de aprendizaje predominantes de los estudiantes en el nivel medio superior en un ambiente mediado por las TIC elaborando cuestionarios. Revista Electrónica del Desarrollo Humano para la 
Innovación Social, $\quad 4(8)$ Recuperado de http://www.cdhis.org.mx/index.php/CAGI/article/view/113/157

Torres, L., \& Rodríguez, N. (2006). Rendimiento académico y contexto familiar en estudiantes universitarios. Enseñanza e investigación, 11(2), 255 - 270.

Varela, M. (2006). Estilo de aprendizaje. Mensaje Bioquímico. XXX, 1 - 11. Recuperado de: https://www.u-cursos.cl/plataforma/2017/1/VA-01-

0250/1/material_docente/previsualizar?id_material $=1680625$

Vinyamata, E. (2011). The Neuro-Linguistic Programming Approach to Conflict Resolution, Negotiation and Change. Journal of Conflictology, 2(1). Recuperado de: http://journal-ofconflictology.uoc.edu/joc/ca/index.php/journal-of-conflictology/article/view/vol2iss1vinyamata.html 
1. Está ayudando a una persona que desea ir al aeropuerto, al centro de la ciudad o a la estación del ferrocarril. Ud.:
a. Iría con ella
b. Le diría como llegar
c. Le daría las indicaciones por escrito (sin mapa)
d. Le daría un mapa

3. No está seguro si una palabra se escribe como "trascendente" $\mathrm{o}$ "tracendente", Ud.:
a. Vería las palabras en su mente y elegiría la que mejor luce.
b. Pensaría en cómo suena cada palabra y elegiría la que mejor luce
c. La buscaría en un diccionario.
d. Escribiría amas palabras y elegiría una

5. Está a punto de comprar una cámara digital o un teléfono móvil. ¿Además del precio, qué más influye en su decisión?

a. Lo utiliza o lo aprueba

b. La lectura de los detalles acerca de las características del equipo.

c. El diseño del equipo es moderno y parece bueno.

d. Los comentarios del vendedor acerca de las características del equipo.

7. Recuerde la vez cuando aprendió cómo hacer algo nuevo. Evite elegir una destreza física, como montar bicicleta. ¿Cómo aprendió mejor?:

a. Viendo una demostración

b. Escuchando la explicación de alguien y haciendo preguntas.

c. Siguiendo pistas visuales en diagramas y gráficas.

d. Siguiendo instrucciones escritas en un manual o libro de texto.

9. Tiene un problema con su rodilla. Preferiría que el doctor:
a. Le diera una dirección web o algo para leer sobre el asunto.
b. Utilizará el modelo plástico de una rodilla para mostrarle qué está mal.
c. Le describiera qué está mal.

2. Está planeando unas vacaciones para un grupo de personas y desearía la retroalimentación de ellos sobre el plan. Ud.:

a. Describiría algunos de los atractivos del viaje

b. Utilizaría un mapa o un sitio web para mostrar los lugares.

c. Les daría una copia del itinerario impreso.

d. Les llamaría por teléfono, les escribiría o les enviaría un e-mail.

4. Va a cocinar algún platillo especial para su familia. Ud.:
a. Cocinaría algo que conoce sin la necesidad de instrucciones.
b. Pediría sugerencias a sus amigos.
c. Hojearía un libro de cocina para tomar ideas de las fotografías.
d. Utilizaría un libro de cocina donde sabe que hay una buena receta.

6. Un grupo de turistas desea aprender sobre los parques o las reservas de vida salvaje en su área. Ud.:
a. Les daría una plática acerca de parques o reservas de vida salvaje.
b. Les mostraría figuras de Internet, fotografías o libros con imágenes.
c. Los llevaría a un parque o reserva y daría una caminata con ellos.
d. Les daría libros o folletos sobre parques o reservas de vida salvaje.

8. Además del precio, ¿qué influiría más en su decisión de comprar un nuevo libro de no ficción?
a. La apariencia le resulta atractiva.
b. Una lectura rápida de algunas partes del libro
c. Un amigo le habla del libro y se lo recomienda
d. Tiene historias, experiencias y ejemplos de la vida real.

10. Está utilizando un libro, CD o sitio web para aprender cómo tomar fotografías con su nueva cámara digital. Le gustaría tener:
a. La oportunidad de hacer preguntas y que le hablen sobre la cámara y sus características. 


\begin{tabular}{|c|c|}
\hline $\begin{array}{l}\text { d. Le mostrara con un diagrama qué } \\
\text { es lo que está mal. }\end{array}$ & $\begin{array}{l}\text { b. Instrucciones escritas con claridad, con } \\
\text { características y puntos sobre qué hacer. } \\
\text { c. Diagramas que muestren la cámara y qué } \\
\text { hace cada una de sus partes. } \\
\text { d. Muchos ejemplos de fotografías buenas y } \\
\text { malas y cómo mejorar éstas. }\end{array}$ \\
\hline $\begin{array}{l}\text { 11. Desea aprender un nuevo programa, } \\
\text { habilidad o juego de computadora. Ud. } \\
\text { debe: } \\
\text { a. Viendo una demostración } \\
\text { b. Escuchando la explicación de } \\
\text { alguien y haciendo preguntas. } \\
\text { c. Siguiendo pistas visuales en } \\
\text { diagramas y gráficas. } \\
\text { d. Siguiendo instrucciones escritas en } \\
\text { un manual o libro de texto. }\end{array}$ & $\begin{array}{l}\text { 12. Prefiere a un profesor o un expositor que } \\
\text { utiliza: } \\
\text { a. Demostraciones, modelos o sesiones } \\
\text { prácticas. } \\
\text { b. Preguntas y respuestas, charlas, grupos } \\
\text { de discusión u oradores invitados. } \\
\text { c. Folletos, libros o lecturas. } \\
\text { d. Diagramas, esquemas o gráficas. }\end{array}$ \\
\hline $\begin{array}{l}\text { 13. Le gustan los sitios web que tienen: } \\
\text { a. Cosas que se pueden picar, mover o } \\
\text { probar. } \\
\text { b. Un diseño interesante y } \\
\text { características visuales. } \\
\text { c. Descripciones escritas interesantes, } \\
\text { características y explicaciones. } \\
\text { d. Canales de audio para oír música, } \\
\text { programas o entrevistas. }\end{array}$ & $\begin{array}{l}\text { 14. Ha acabado una competencia o una prueba y } \\
\text { quisiera una retroalimentación. Quisiera } \\
\text { tener la retroalimentación: } \\
\text { a. Utilizando ejemplos de lo que ha hecho. } \\
\text { b. Utilizando una descripción escrita de sus } \\
\text { resultados. } \\
\text { c. Escuchando a alguien haciendo una } \\
\text { revisión detallada de su desempeño. } \\
\text { d. Utilizando gráficas que muestren lo que } \\
\text { ha conseguido. }\end{array}$ \\
\hline $\begin{array}{l}\text { 15. Va a elegir sus alimentos en un } \\
\text { restaurante o café. Ud.: } \\
\text { a. Elegiría algo que ya ha probado en } \\
\text { ese lugar. } \\
\text { b. Escucharía al mesero o pediría } \\
\text { recomendaciones a sus amigos. } \\
\text { c. Elegiría a partir de las } \\
\text { descripciones del menú. } \\
\text { d. Observaría lo que otros están } \\
\text { comiendo o las fotografías de cada } \\
\text { platillo. }\end{array}$ & $\begin{array}{l}\text { 16. Tiene que hacer un discurso importante para } \\
\text { una conferencia o una ocasión especial. Ud.: } \\
\text { a. Elaboraría diagramas o conseguiría } \\
\text { gráficos que le ayuden a explicar las } \\
\text { ideas. } \\
\text { b. Escribiría algunas palabras clave y } \\
\text { práctica su discurso repetidamente. } \\
\text { c. Escribiría su discurso y se lo aprendería } \\
\text { leyéndolo varias veces. } \\
\text { d. Conseguiría muchos ejemplos e historias } \\
\text { para hacer la charla real y práctica. }\end{array}$ \\
\hline
\end{tabular}

